\title{
VLA polarimetry of two extended radio galaxies
}

\author{
W. Junor ${ }^{1}$, F. Mantovani ${ }^{2}$, R. Morganti ${ }^{2,3}$, and L. Padrielli ${ }^{2}$ \\ 1 Institute for Astrophysics, University of New Mexico, Albuquerque, NM, U.S.A. \\ 2 Istituto di Radioastronomia del CNR, Bologna, Italy \\ 3 Australian Telescope National Facility, Epping, NSW 2121, Australia
}

Received May 7, 1996; accepted February 25, 2000

\begin{abstract}
Multi-wavelength VLA observations of two extended radio galaxies, $0235-197$ and $1203+043$ are presented. There is some evidence from earlier studies that these two sources exhibit low frequency $(<1 \mathrm{GHz})$ variability. This work shows that both sources have linear polarizations, if any, below the detection limits at $320 \mathrm{MHz}$, so we cannot explain the variability as being due to instrumental polarization effects as has been suggested for 3C 159. Refractive scintillation may be the cause of the variability in $0235-197$. This would require the existence of a bright, compact component in one of the hot spots seen in these observations. This is not implausible but the resolution of this observational program is insufficient to address that question. The radio source $1203+043$ lacks any bright compact component thereby ruling out a refractive scintillation mechanism for its variability. Consequently, it is possible that claims of variability in this source are spurious. However, the $320 \mathrm{MHz}$ VLA observations show that $1203+043$ has an "X"-shaped radio structure. This is a rare morphology for the brightness distribution of a radio galaxy; the implications of this are examined.
\end{abstract}

Key words: galaxies: individual: $0235+197,1203+043$ techniques: polarimetric — radio continnum: galaxies

\section{Introduction}

As part of our investigation of steep-spectrum $\left(\alpha>0.5, S \propto \nu^{-\alpha}\right)$, low-frequency-variable (LFV; $\nu<$ $1 \mathrm{GHz}$ ) sources, we have made a series of images with sub-arcsecond resolutions (Mantovani et al. 1992) of a sample of sources. These sources were selected from the papers of Cotton (1976), McAdam (1980), Spangler \& Cotton (1981), Fanti et al. (1983) and Altschuler

Send offprint requests to: F. Mantovani e-mail: fmantovani@ira.bo.cnr.it et al. (1984). The aim was to detect the high-brightness components required by the refractive scintillation model for low-frequency variability (Rickett 1986). Most of the sources in the sample showed compact features (deconvolved sizes $<0.15-0.3$ arcsec) both in MERLIN, $408 \mathrm{MHz}$ and VLA, A-array, $5 \mathrm{GHz}$ images (Mantovani et al. 1992). Further observations with VLBI show these features to contain components which are bright and compact enough to explain the variability at low frequency by propagation effects in the interstellar medium; see, for example, 3C 99, Mantovani et al. (1990a). Sources such as these do not generally show any variability at high frequency (Padrielli et al. 1987).

However, there are sources like $0621+400$ (3C 159), which are variable at low frequencies and not at frequencies $>2 \mathrm{GHz}$, where the compact components are too weak to account for the observed variability. In these cases, it is possible that the variability is caused by instrumental effects. The source 3C 159 has been monitored for about 10 years at $408 \mathrm{MHz}$. This source has a steep radio spectrum and an extended double radio structure - a combination which is very unusual for a variable radio source. Browne et al. (1985) have suggested that the variations, which seem to show an annual cycle, may not be intrinsic but could arise from the combined effects of strong source linear polarization and ionospheric Faraday rotation.

Ionospheric Faraday rotation can easily reach $7-8 \mathrm{rad} \mathrm{m}^{-2}$ (Sakurai \& Spangler 1994). With the plausible estimate of $5 \mathrm{rad} \mathrm{m}^{-2}$ as an expectable difference in the ionospheric RM, one finds that the position angle difference at $408 \mathrm{MHz}$ is 2.7 radians; enough to produce the effect being discussed.

MERLIN observations at $408 \mathrm{MHz}$ (Cerchiara et al. $1994)$ show that $3 \mathrm{C} 159$ is highly polarized $(\sim 10 \%)$. The plane of polarization of the source emission could be rotated by changes in ionospheric Faraday rotation relative to the linearly polarized E-W arm of the Northern Cross Bologna telescope used for the monitoring program. A source with a linear polarization $>6 \%$ could exhibit 
Table 1. VLA observing dates

\begin{tabular}{|l|r|c|c|c|}
\hline Source & $\begin{array}{r}\text { Band } \\
\text { MHz }\end{array}$ & Array & $\begin{array}{c}\text { Duration } \\
\text { minutes }\end{array}$ & $\begin{array}{c}\text { Observing } \\
\text { Date }\end{array}$ \\
\hline \hline $0235-197$ & 320 & A & 87 & 22 Dec. 1992 \\
& 4885 & A & 30 & 30 May 1986 \\
& 4885 & C & 15 & 27 May 1989 \\
& 8440 & A & 49 & 10 Sep. 1990 \\
& 14940 & A & 51 & 10 Sep. 1990 \\
$1203+043$ & 320 & A & 67 & 09 Dec. 1992 \\
& 4885 & A & 39 & 30 May 1986 \\
& 8440 & A & 28 & 10 Sep. 1990 \\
& 14940 & A & 32 & 10 Sep. 1990 \\
\hline
\end{tabular}

apparent variations of roughly the observed size if the ionospheric Faraday rotation changed by $\sim 90^{\circ}$ between observations. The 3C 159 observations were made at transit during the day in summer and the night in winter and so they were accompanied by annual changes in the ionospheric electron content.

The purported variability measured in the two extended radio sources $0235-197$ and $1203+043$ may have originated in a similar manner to that in 3C 159. They were monitored at $408 \mathrm{MHz}$ with a similar instrument, the Molonglo Cross. With a peak-to-peak fractional variability of $\sim 10 \%$, they were classified as "probably variable" by McAdam (1980).

Consequently, although it was expected that most of the sources belonging to our sample of steep-spectrum, low-frequency-variable sources would be core-dominated sources, it is likely that the sample has been contaminated by lobe-dominated, strongly-linearly-polarized, extended sources.

In order to test if ionospheric Faraday rotation is the cause of the apparent variability of $0235-197$ and $1203+043$ we have investigated the linear polarizations of these sources at $320 \mathrm{MHz}$ with the VLA in the "A" configuration and with the already available $5 \mathrm{GHz}$ VLA C-array data. Both sources were also observed in the $X(8.4 \mathrm{GHz})$ and $U(15 \mathrm{GHz})$ bands. These observations allowed high resolution images of the "hot spot" regions to be made. The images were combined with available, high-resolution, $C$ band observations to produce rotation measures (RMs) for the outer parts of the sources.

\section{VLA observations}

VLA (Thompson et al. 1980) observing dates and observational parameters are summarized in Table 1 . The high resolution (A-array) Total Intensity images at $5 \mathrm{GHz}$ for 0235-197 and 1203+043 were presented in Mantovani et al. (1992) while the lower resolution C-array, total intensity map for $0235-197$ has been published in Morganti et al. (1993). However, because the polarization information was never presented in the earlier papers, we have summarized in the tables all the observational details and the derived parameters from those observations. Because of narrowband interference, the $P$
$(320 \mathrm{MHz})$ band data were taken in spectral line mode. The data were edited to remove channels with interference. Bandpass corrections were determined from the calibrator source 3C $286(1331+305)$ and applied to the spectral line database. A new "Channel 0" database was then constructed and the data were calibrated for total intensity and polarization in the standard fashion (see, for example, Perley et al. 1989). Instrumental polarization calibration was done using the calibration sources 3C 48 $(0134+329), 3 \mathrm{C} 138(0521+166)$ and $3 \mathrm{C} 286(1331+305)$ to get sufficient parallactic coverage across both days during which the target sources were observed. We assume that the instrument is stable between observing epochs.

The parallel hand (RR, LL) data were self-calibrated and imaged in the normal iterative manner. The complex gain corrections derived from self-calibration were also applied to the cross-hand (RL or LR) fringes. In turn, images in Stokes parameters $I, Q, U$ and $V$ were produced. Maps of the polarized flux density $P=\left(Q^{2}+U^{2}\right)^{1 / 2}$ and position angle $\chi=0.5 \times \tan ^{-1}(U / Q)$, were then generated from the $Q$ and $U$ images. The Stokes $V$ images were used to test the integrity of the calibration and self-calibration procedures and to diagnose problems due to interference.

At low frequencies, the primary beam of the antennas contains many background sources. In order to image the target sources of interest, it was necessary to image some of these background sources. The brightest ( $>20 \mathrm{mJy}$ at $L$ band) background sources were identified from the NRAO VLA Sky Survey (NVSS) (Condon et al. 1998). This threshold is somewhat arbitrary but gives a reasonable number of secondary fields to image at $320 \mathrm{MHz}$. We imaged a total of 26 fields for $0235-197$ and 23 fields for $1203+043$ in the AIPS mapping program, IMAGR - one field containing the program source and the others on the brightest background sources. We were able to account for substantially all of the flux density seen on the shortest baselines in both cases and to obtain satisfactory convergence in the iterative self-calibration and imaging loop. The final images in all Stokes parameters $(I, Q, U \& V)$ of the target sources contained no obvious artefacts due to sidelobes from nearby confusing sources. The linear polarizations of the background sources have been checked in order to test for beam squint between $R$ and $L$ beams. The rms noises in the final images are within a factor of 3 of the expected thermal noises; this is entirely consistent with other observing programs at $320 \mathrm{MHz}$. Together, these suggest that the images of the target sources are not greatly affected by confusion.

The low declination source 0235 - 197 was observed for only $\approx 2$ hours at transit; consequently, some of the data were corrupted by cross-talk between antennas. These data, mostly on baselines with immediately adjacent antennas, were excised from the database during the iterative self-calibartion and imaging process. 


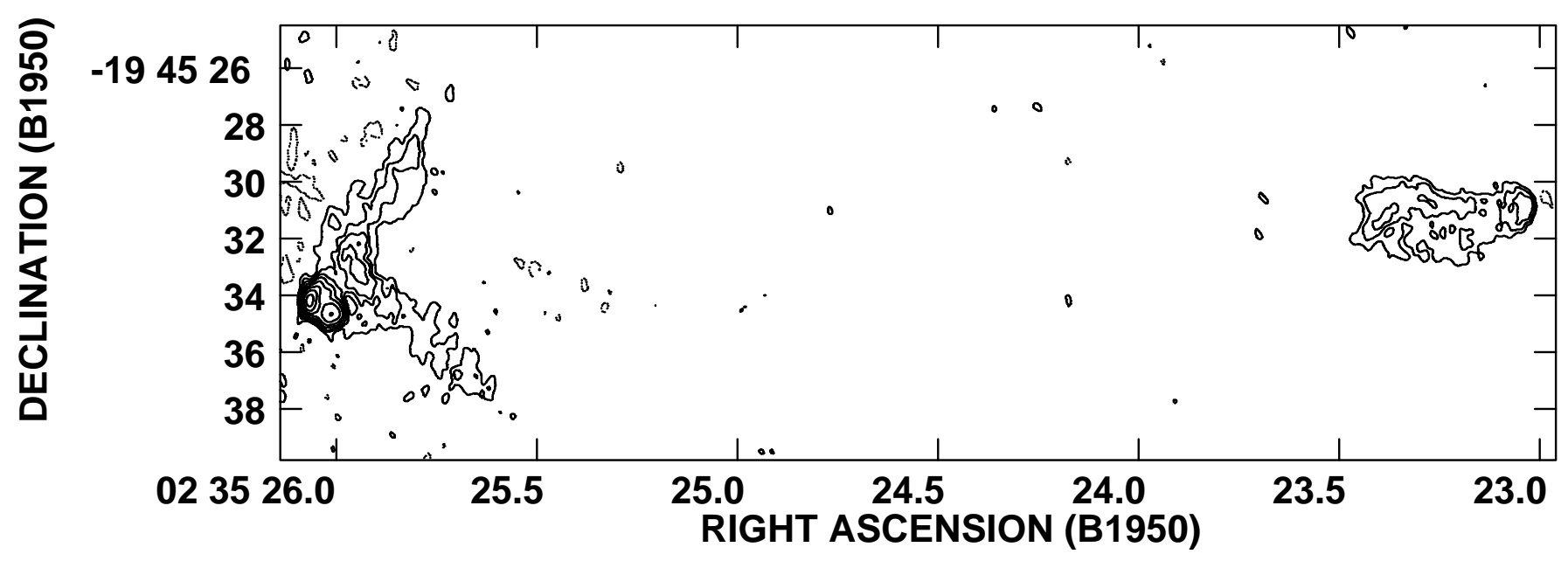

Fig. 1. VLA image of $0235-197$ at $8.4 \mathrm{GHz}$. Contours are at $-0.3,0.3,0.6,1,2,4,8,16,32,64 \mathrm{mJy}^{\text {beam }}{ }^{-1}$. The peak flux density is $45.4 \mathrm{mJy}_{\text {beam }}{ }^{-1}$

The $C(5 \mathrm{GHz}), X(8.4 \mathrm{GHz})$ and $U(15 \mathrm{GHz})$ band data were calibrated in the standard way using VLA calibrators and AIPS procedures. Polarimetric images were generated in a manner similar to that for the $P$ band data.

\section{Sources structure and polarimetry}

\subsection{Observational parameters}

The derived parameters for the low resolution observations at $320 \mathrm{MHz}$ and at $5 \mathrm{GHz}$ (VLA C-array) are listed in Table 2. Maps at higher resolution have been obtained at 8.4 and $15 \mathrm{GHz}$ with the VLA in the A configuration. Values derived from the maps are listed in Table 3. Comments on the sources structure will be given in Sect. 4 .

The contents of Tables 2 and 3 are: Col. 1 - source name; Col. 2 - the observing frequency in MHz; Cols. 3 to 5 - major axis, minor axis (both in arcsec) and the PA in degrees of the restoring beam major axis; Col. 6 - the rms noise in the total intensity map far from the source of emission; Col. 7 - the rms noise $\sqrt{\sigma_{Q}^{2}+\sigma_{U}^{2}}$, where $\sigma_{Q}$ and $\sigma_{U}$ are the rms noises on the blank sky in the distributions of the Stokes parameters $Q$ and $U$; Col. 8 component label; Cols. 9 and 10- RA and Dec. of the component peak; Col. 11 - peak flux density (mJy) of the component; Col. 12 - total flux density (mJy) of the component.

In Tables 4 and 5, we give the measured position angle (PA) in degrees of the electric field vector at the peak of polarized emission ( $\pm 1 \mathrm{rms}$ error calculated from the distribution of PAs found in a small box around the peak of polarized emission); the Rotation Measure $\left(R M=\Delta \phi(\lambda) \pm n \pi / \Delta\left(\lambda^{2}\right)\right.$ in $\mathrm{rad} \mathrm{m}^{-2}$ where $\phi(\lambda)$ is the PA at wavelength $\lambda$ and $n$ an integer; when three frequencies are available, as for some of the components in Table 4 , the ambiguity inplied by the integer $n$ can be resolved); the $R M$, corrected for the redshift; the percentage polarization; the depolarization index, defined as the ratio of the fractional polarization at longer wavelength to the fractional polarization at shorter wavelength; from the high and low resolution observations.

In order to compare the $5 \mathrm{GHz}$ (C-Array) and $320 \mathrm{MHz}$ images of $0235-197$, we have produced $5 \mathrm{GHz}$ maps ( $I$, $Q, U)$ at the resolution of the $320 \mathrm{MHz}$ maps. This was done by restoring the $5 \mathrm{GHz}$ images with the appropriate Gaussian beam during imaging. The polarization parameters derived from those images are reported in Table 5.

\section{Notes on individual sources}

4.1. $0235-197$

The $5 \mathrm{GHz}$ images presented by Mantovani et al. (1992) and Morganti et al. (1993), show the classical, double structure typical of powerful radio galaxies. The source is associated with a galaxy at $z=0.620$ (Tadhunter et al. 1993). There are no radio components above the detection limits $(\leq 0.2 \mathrm{mJy}$ at 5 and $8.5 \mathrm{GHz} ; \leq 0.5 \mathrm{mJy}$ at $15 \mathrm{GHz}$ ) inside the error box of the optical position. Adopting the optical position as a reference, 0235-197 looks rather symmetric with a ratio of $\sim 0.8$ between the full length of the two lobes, with the western being the longer.

0235-197 appears to be dominated by the outer lobes at frequencies $>5 \mathrm{GHz}$. The most interesting feature is the bright hot spot at the far end of the Eastern lobe. At both 5 and $8.4 \mathrm{GHz}$ (Figs. 1 to 3), the hot spot has a double structure with individual components labelled $E_{1}$ and $E_{2}$. Component $E_{1}$ also appears double when observed with higher resolution at $15 \mathrm{GHz}$ (Fig. 4). The images have been convolved to 0.4 arcsec resolution in order to calculate the hot spot spectral indices. Both components $E_{1}$ and $E_{2}$ show a large steepening in spectral index, $\alpha\left(S \propto \nu^{-\alpha}\right)$, between 
Table 2. Low resolution observational parameters and observed properties

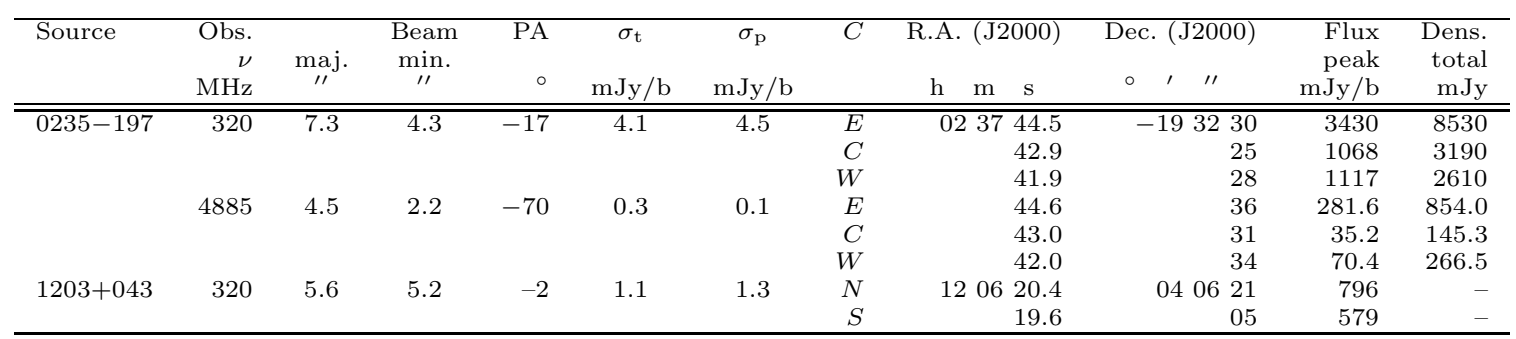

Table 3. High resolution observational parameters and observed properties

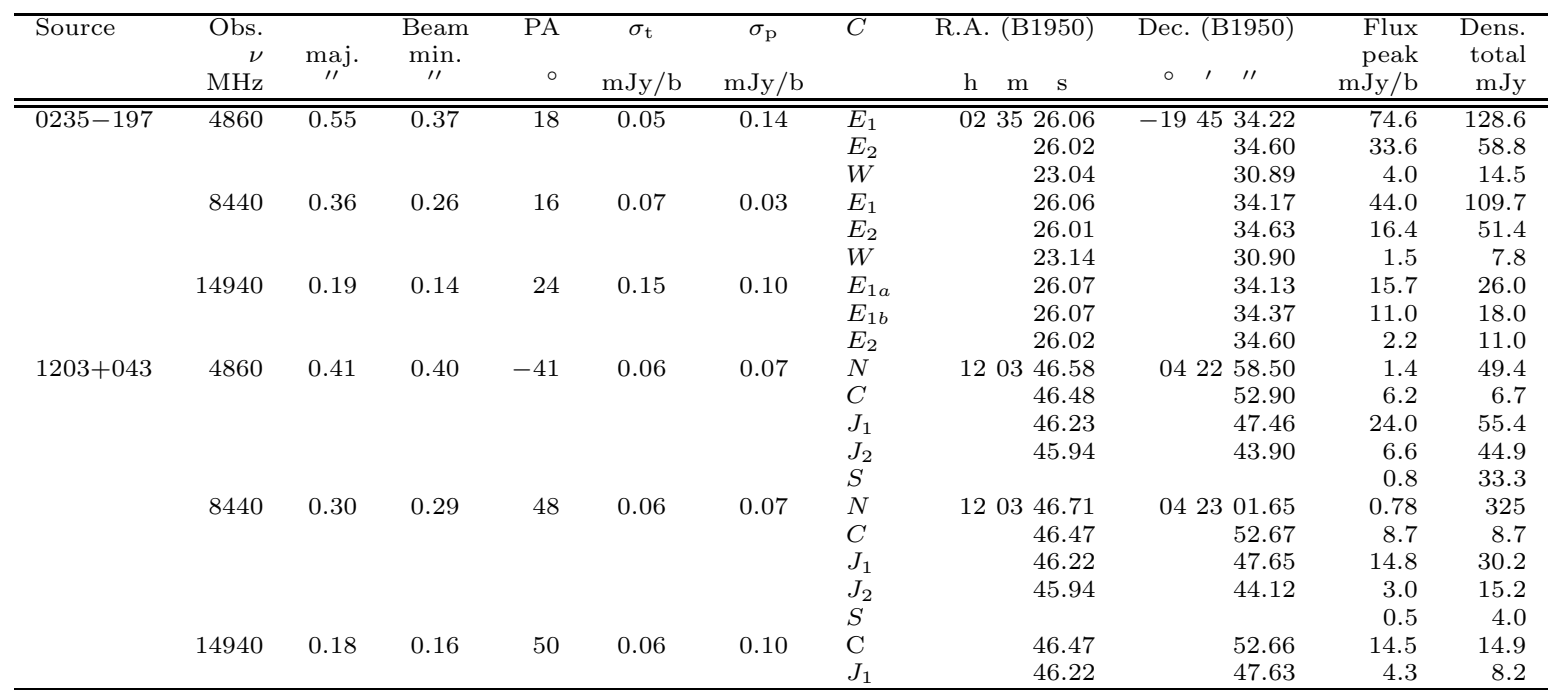

$5-8.4 \mathrm{GHz}$ and $8.4-15 \mathrm{GHz}$. We find values of $\alpha=0.5$ and $\alpha=1.4$ respectively for $E_{1}$ and $\alpha=0.68$ and $\alpha=2.5$ respectively for $E_{2}$. The front shock of the lobe $W_{1}$ at the opposite side is resolved in all of the images and can hardly be defined as a "hot spot". The spectral index of the bright part at the far end is also steep $(\alpha=1.2)$ in the range $5-8.4 \mathrm{GHz}$ and it steepens to $\alpha \geq 2$ in the range $8.4-15 \mathrm{GHz}$ (since the $15 \mathrm{GHz}$ flux density estimate is an upper limit). Note that the observations at $15 \mathrm{GHz}$ have lower sensitivity to diffuse, extended emission.

All of the hot spots are highly polarized, with little depolarization and Faraday rotation. The magnetic field is parallel to the front shock and rather ordered in the lobe regions with weak diffuse emission. (The electric vector is shown in all of the images shown in the paper). At $320 \mathrm{MHz}$ (Fig. 5), the polarized emission, if any, is below the detection limit of our observations. Polarized emission is detected over all of the source in the low resolution observations at $5 \mathrm{GHz}$ (Fig. 6). The magnetic field is again ordered and, generally speaking, parallel to the source major axis, apart from the hot spot area, where the magnetic field is parallel to the front shock. There are two main regions of polarized emission in the $E$ lobe. The mean PA given in Table 5 should be treated with care because the position angles of the polarization vectors in the lobe actually vary greatly. The depolarization between $5 \mathrm{GHz}$ and $320 \mathrm{MHz}$ is very high $(D P>0.02)$. Statistically, in these classical sources, the lobe nearest to the nucleus usually shows a steeper spectral index. Here we find similar values $(\alpha=0.84)$ for the two lobes of $0235-197$. There are indications of Faraday rotation in the hot spots from the high resolution maps.

\section{2. $1203+043$}

The images at 8.4 and $15 \mathrm{GHz}$ (Figs. 7 to 8 and 9 respectively) do not add much new information about the overall source structure as derived from the $5 \mathrm{GHz}$ image of Mantovani et al. (1992). This earlier image showed a long bent jet. The components found along the jet, labelled $J_{1}$ and $J_{2}$, are rather polarized. The emission from $J_{1}$ has a small Faraday rotation and depolarization between $6 \mathrm{~cm}$ and $4 \mathrm{~cm}$ of $17 \mathrm{rad} \mathrm{m}^{-2}$ and 0.74 respectively.

However, the new observations have allowed us to identify component $C$ with the core of the radio source. This component has an inverted spectrum which peaks at frequencies $\geq 15 \mathrm{GHz}$. $1203+043$ therefore has an asymmetric structure, with a long bent jet pointing South which fades slowly and with a weak lobe of emission to the north 
Table 4. Polarization parameters from high resolution observations

\begin{tabular}{|c|c|c|c|c|c|c|c|c|c|c|c|c|}
\hline \multirow[t]{2}{*}{ Source } & \multirow[t]{2}{*}{$z$} & \multirow[t]{2}{*}{$C$} & \multicolumn{3}{|c|}{$\mathrm{PA}$} & \multirow[t]{2}{*}{$R M$} & \multirow{2}{*}{$\begin{array}{r}R M \times \\
(1+z)^{2}\end{array}$} & \multicolumn{3}{|c|}{$\% \mathrm{Pol}$} & \multirow{2}{*}{$\begin{array}{r}D P \\
6-4\end{array}$} & \multirow{2}{*}{$\begin{array}{r}D P \\
4-2\end{array}$} \\
\hline & & & $6 \mathrm{~cm}$ & $4 \mathrm{~cm}$ & $2 \mathrm{~cm}$ & & & $6 \mathrm{~cm}$ & $4 \mathrm{~cm}$ & $2 \mathrm{~cm}$ & & \\
\hline \multirow[t]{3}{*}{ "0235-197 } & 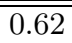 & $\overline{\overline{E_{1}}}$ & $\overline{68 \pm \pm 6}$ & $\overline{77 \pm 2}$ & 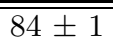 & $\overline{87}$ & $\overline{2228}$ & $\overline{\overline{22.0}}$ & 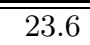 & $\overline{20.2}$ & 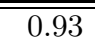 & $\overline{\overline{1.2}}$ \\
\hline & & $E_{2}$ & $-80 \pm 7$ & $-84 \pm 2$ & $82 \pm 2$ & -87 & -228 & 13.4 & 17.8 & 8.4 & 0.75 & 2.1 \\
\hline & & $W_{1}$ & $78 \pm 4$ & $83 \pm 2$ & - & 35 & 92 & 22.8 & 19.5 & - & 1.20 & - \\
\hline \multirow[t]{2}{*}{$1203+043$} & & $J_{1}$ & $68 \pm 3$ & $62 \pm 1$ & $66 \pm 6$ & 17 & & 15.0 & 20.2 & 5.5 & 0.74 & 3.7 \\
\hline & & $J_{2}$ & $-68 \pm 8$ & $-49 \pm 1$ & - & 140 & & 15.8 & 10.9 & - & 1.45 & . \\
\hline
\end{tabular}

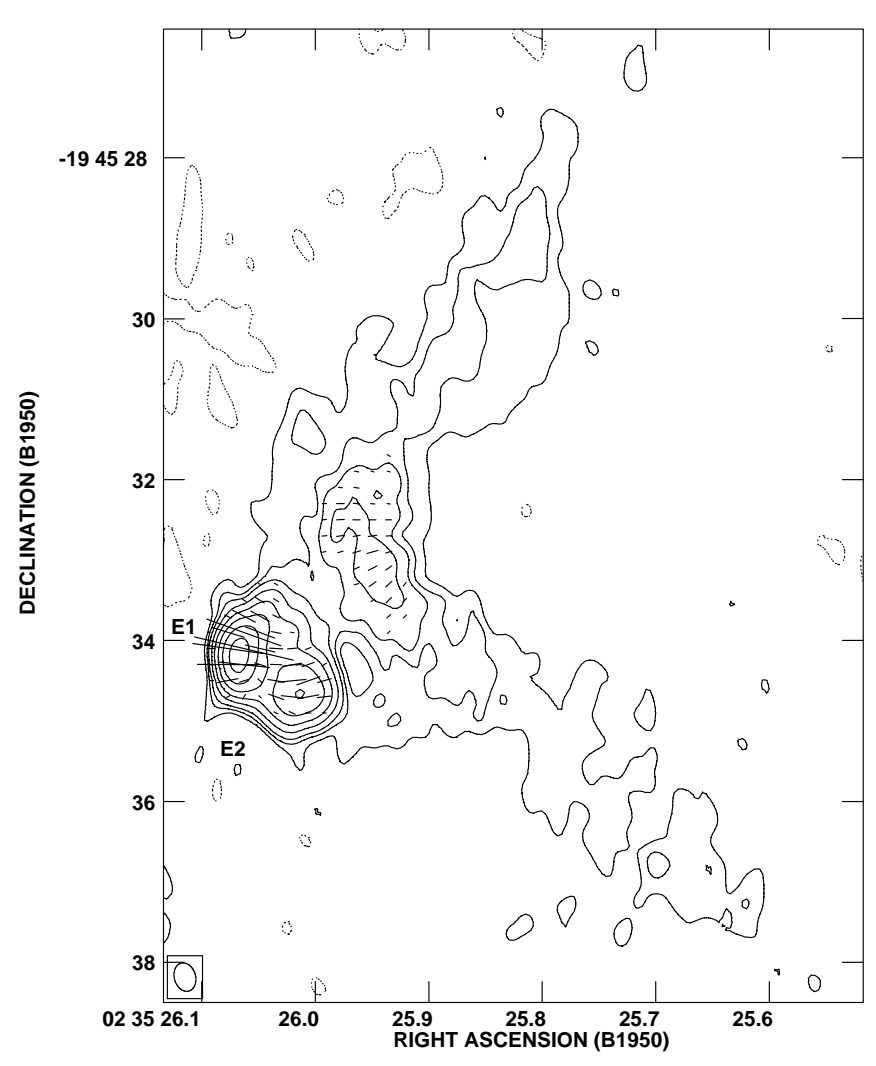

Fig. 2. VLA image of the East lobe of $0235-197$ at $8.4 \mathrm{GHz}$. Contours are at $-0.3,0.3,0.6,1,2,4,8,16,32,64$ mJy beam $^{-1}$. A vector length of $1^{\prime \prime}=10$ mJy beam $^{-1}$

where there is marginal evidence of an hot spot. The radio position of the core of $1203+043$ does not coincide within the errors to any optical counterpart on the Palomar Sky Survey prints.

Much more interesting is the structure found at $320 \mathrm{MHz}$ (Fig. 10). Together with the North-South structure which dominates at higher frequencies and which appears here as a ridge of emission, there is a region of diffuse emission with its major axis perpendicular to the main ridge. This new feature is about 50 arcsec in extent and is comparable in width with the main North-South ridge.

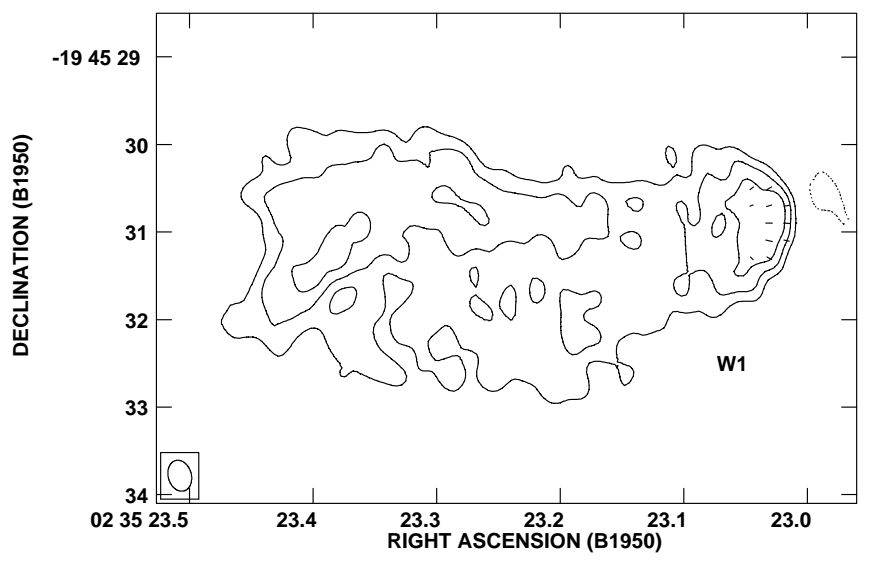

Fig. 3. VLA image of the West lobe of $0235-197$ at $8.4 \mathrm{GHz}$. Contours are at $-0.3,0.3,0.6,1,2,4,8,16,32,64$ mJy beam $^{-1}$. A vector length of $1^{\prime \prime}=10$ mJy beam $^{-1}$

Table 5. Polarization parameters from low resolution observations of $0235-197$ at $6 \mathrm{~cm}$

\begin{tabular}{lccc}
\hline Source & $C$ & PA & \% Pol \\
\hline \hline 0235-197 & $E$ & $-16 \pm 18$ & 10.5 \\
& $C$ & $-85 \pm 1$ & 9.1 \\
& $W$ & $-18 \pm 5$ & 20.6 \\
\hline
\end{tabular}

\section{Discussion}

Due to the non-detection of polarized emission at $320 \mathrm{MHz}$ in both $0235-197$ and $1203+043$, we cannot explain the low frequency variability observed with the Molonglo Cross (McAdam 1980) in terms of ionospheric Faraday rotation as in the case of $3 \mathrm{C} 159$.

Can refractive scintillation (Rickett 1986) explain the variability of $0235-197$ ? The refractive scintillation models assume a supposedly-variable radio source to have most of its flux density in a single compact component. The degree of variability is a function of the characterization of the interstellar medium (itself a function of galactic coordinates) and source size. $0235-197$ is at galactic latitude $|b|=65^{\circ}$ and was reported to vary (rms variability $\sim 0.2 \mathrm{Jy}$ ) on a time scale of the order of 1 year (McAdam 1980). 


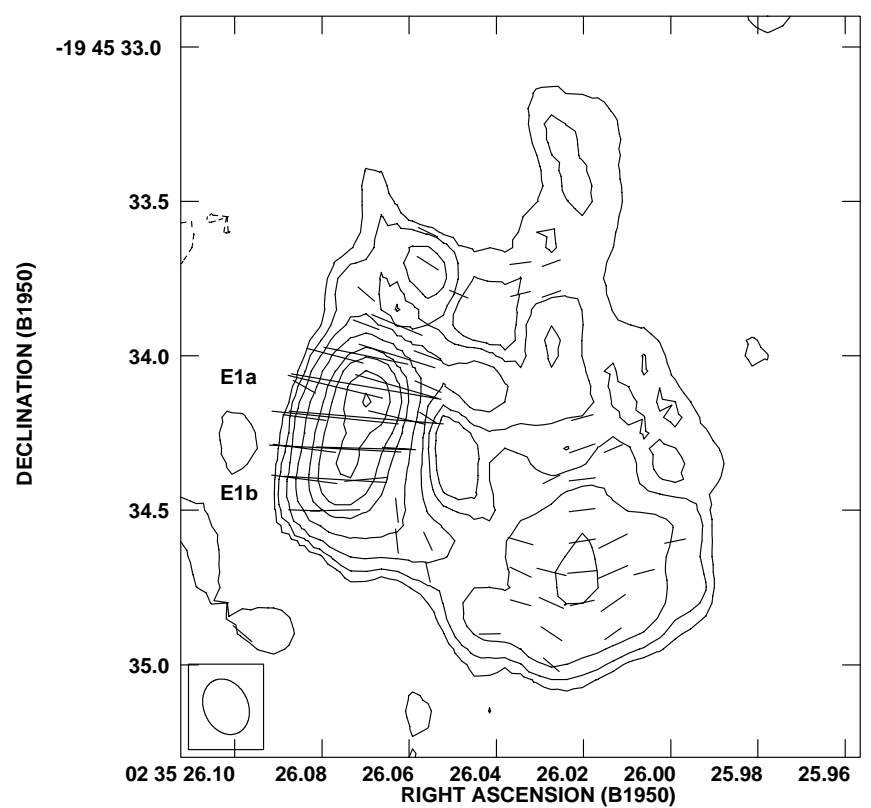

Fig. 4. VLA image of the East lobe of $0235-197$ at $15 \mathrm{GHz}$. Contours are at $-0.4,0.4,0.6,1,2,4,6,10,15,20$ mJy beam $^{-1}$. The peak flux density is $15.7 \mathrm{mJy}_{\text {beam }}{ }^{-1}$. A vector length of $1^{\prime \prime}=6.7$ mJy beam $^{-1}$

In the usual refractive model of interstellar turbulence (Mantovani et al. 1990b; Spangler et al. 1993; Spangler et al. 1994; Bondi et al. 1994), the relevant parameter for the observed scintillation index is $\theta_{\text {eff }}^{7 / 6} \sqrt{\sin b / I}$, where $\theta_{\text {eff }}=\theta_{\mathrm{FWHM}} / 2.35$ and $I$ is the parameter indicator of the source structure ( $=1$ for a Gaussian structure). In such a model a source of $(\approx 3 \mathrm{Jy})$ with a rms variability of $0.2 \mathrm{Jy}$ and corresponding scintillation index of $\sim 0.06$ should have an angular diameter in the range 10 - 20 mas (see Spangler et al. 1993 for details).

The $E_{1 \mathrm{a}}$ hot spot has a spectrum which, extrapolated towards lower frequencies, gives a mean flux density of $\sim 3 \mathrm{Jy}$ at $408 \mathrm{MHz}$. This is comparable to the peak flux density found at $320 \mathrm{MHz}$. The crucial parameter is, however, the angular size of the hot spot. The deconvolved size found for the $E_{1 \mathrm{a}}$ hot spot at $15 \mathrm{GHz}$ is $<0.2$ arcsec. Even if in principle there is not contradiction, the scintillation theory requires an angular size for the hot spot in 0235-197 that is a factor 7-10 smaller than the size measured at $15 \mathrm{GHz}$, which is close to the sizes usually measured for the hot spots. Low frequency VLBI observations are needed to confirm the existence of such a compact component in the hot spot.

The lack of polarized emission at $320 \mathrm{MHz}$ for $1203+043$ and a radio structure which lacks a bright compact component rules out both of the mechanisms for low frequency variability in this source. Consequently, we

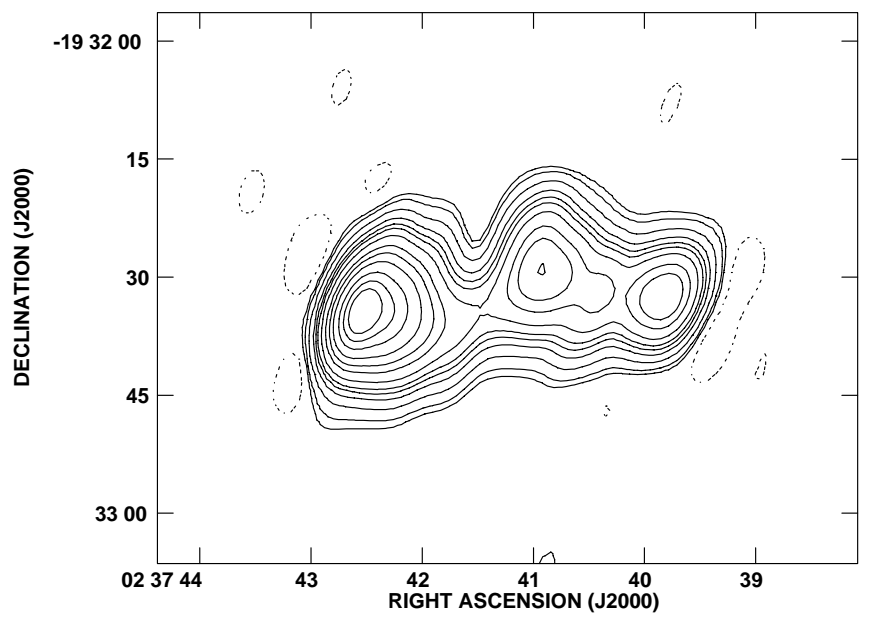

Fig. 5. VLA image of $0235-197$ at $320 \mathrm{MHz}$. Contours are at $-5,5,10,20,50,100,150,200,300,500,700,1000,1500,2000$, $2500 \mathrm{mJy}$ beam $^{-1}$. The peak flux density is $3430 \mathrm{mJy}^{\text {beam }}{ }^{-1}$

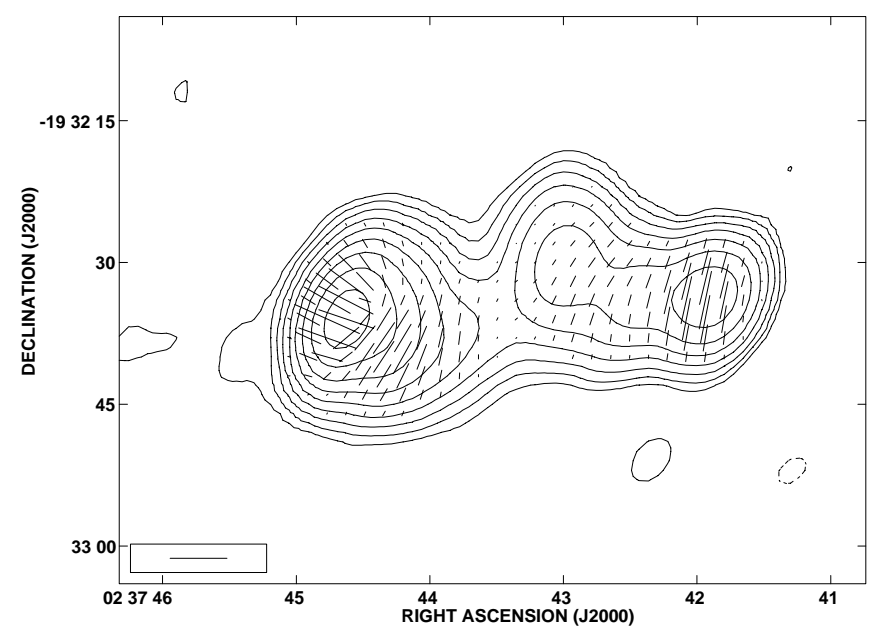

Fig. 6. VLA image of $0235-197$ at $5 \mathrm{GHz}$. Contours are at $1.1 \times\left(-1,1,2,4,8,16,32,64,128,256\right.$ mJy beam $^{-1}$. The peak flux density is 419.1 mJy beam $^{-1}$. A vector length of $1^{\prime \prime}=$ 5 mJy beam $^{-1}$

conclude that this source might be a spurious case of variability. However, $1203+043$ has an interesting structure at $320 \mathrm{MHz}$. It shows a pair of secondary lobes in a direction perpendicular to the main source axis, making the object one of a few known "X"-shaped sources. At present, only about ten sources are known to show such morphology. They are believed to have both young and old lobes. These lobes may be supplied by jets whose direction has changed with time. A change in the orientation of the central engine due to precession has been suggested by Ekers et al. (1978) for NGC 326 to account for its 


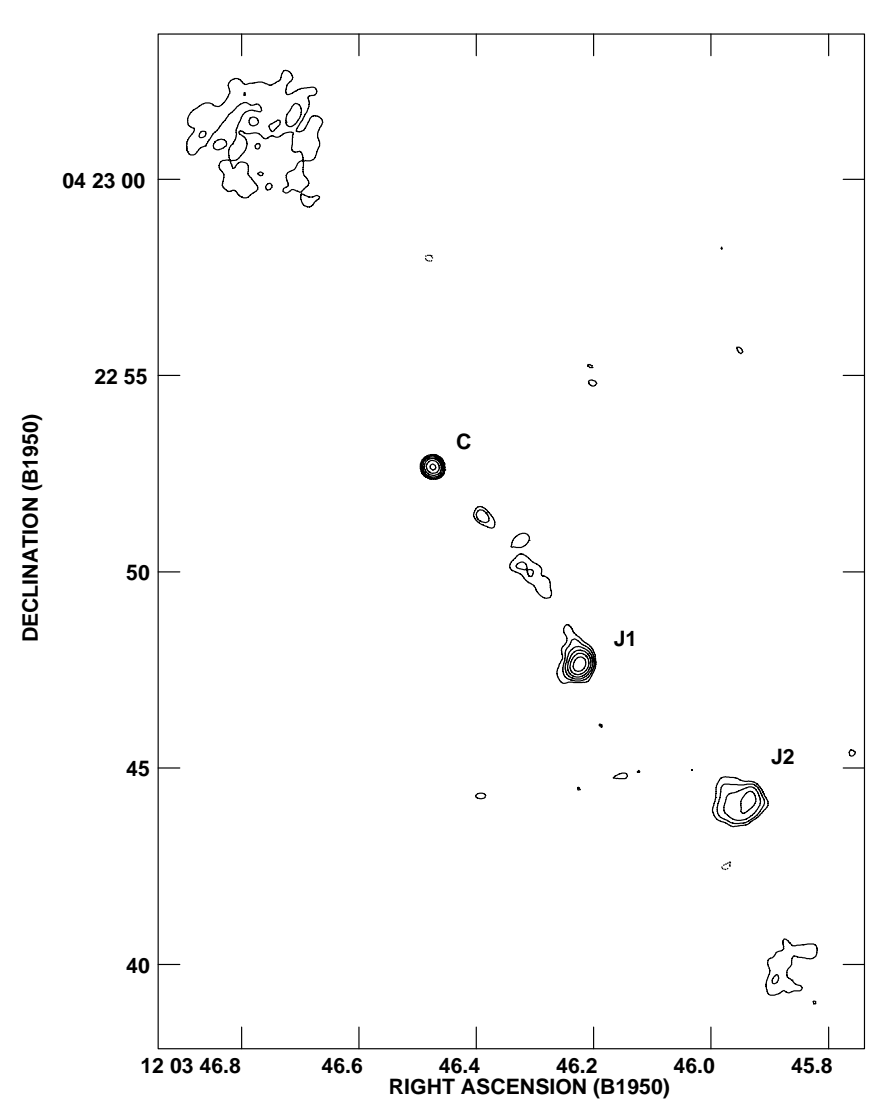

Fig. 7. VLA image of $1203+043$ at $8.4 \mathrm{GHz}$. Contours are at $-0.25,0.25,0.5,1,2,4,8,16,32,64,128$ mJy beam $^{-1}$. The peak flux density is 14.8 mJy beam $^{-1}$

"X" shaped morphology. Such a model has been applied successfully to $0828+32$ by Klein et al. (1995) but with the extra assumption that the length of the precessing beam changes with time. A merger between galaxies is thought to be the cause of the precession. However, Ulrich-Demoulin \& Rönnback (1996) have reported that optical images of $0828+32$ do not show the signature of a recent major merger event.

However, the structure of $1203+043$ looks peculiar when compared with other "X" shaped sources. For example, it has an asymmetric structure with respect to the component $C$ which is believed to be the core (Figs. 7 and 6 in Mantovani et al. 1992). The long, bent jet is clearly "one-sided" while, generally speaking, the members of the class show two-sided jets (at the available resolution). Moreover, the young lobes of the "X" shaped sources are dominated by hot spots while here the jet emission fades away from the core and the northern lobe contains only diffuse emission without any bright component. This asymmetry is reflected in the $320 \mathrm{MHz}$ map where the region to the North-West is more extended and brighter than the opposite side.

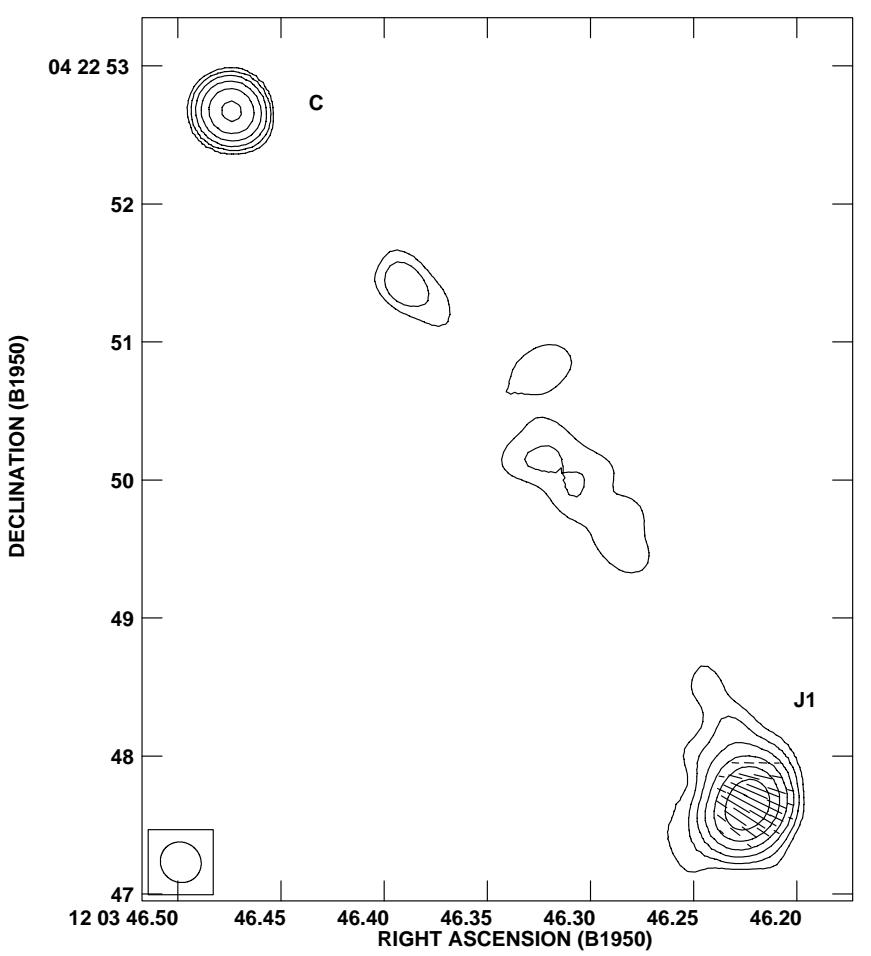

Fig. 8. VLA image of the central region of $1203+043$ at $8.4 \mathrm{GHz}$. Contours are at $-0.25,0.25,0.5,1,2,4,8,16,32,64$, $128 \mathrm{mJy}$ beam $^{-1}$. The peak flux density is $14.8 \mathrm{mJy} \mathrm{beam}^{-1}$. A vector length of $1^{\prime \prime}=10 \mathrm{mJy}_{\text {beam }}{ }^{-1}$

\section{Conclusions}

We have conducted a program of multi-wavelength VLA observations of the suspected low frequency variable sources $0235-197$ and $1203+043$. Since $0235-197$ is not polarized at $320 \mathrm{MHz}$, its variability cannot be accounted for by instrumental polarization effects as in the case of 3C 159. 0235-197 may contain a low frequency component sufficiently compact and bright as required by the refractive scintillation model for low frequency variability. Our observations have insufficient resolution to test this suggestion; low frequency VLBI observations are required for this purpose. However, this component would have to have extremely unusual properties among hot spots in radio sources.

In our high frequency images of $1203+043$ we have identified the core of the radio source; its location indicates that the source has a large apparent asymmetry. At $320 \mathrm{MHz}$, this source shows no polarization. However, it does have an additional, steep-spectrum component at this frequency; this previously-undetected component lies perpendicular to the main axis and predominantly to one side. However, the overall morphology of $1203+043$ at low frequencies seems similar to that of the "X"shaped sources like NGC 326. From its morphology and 


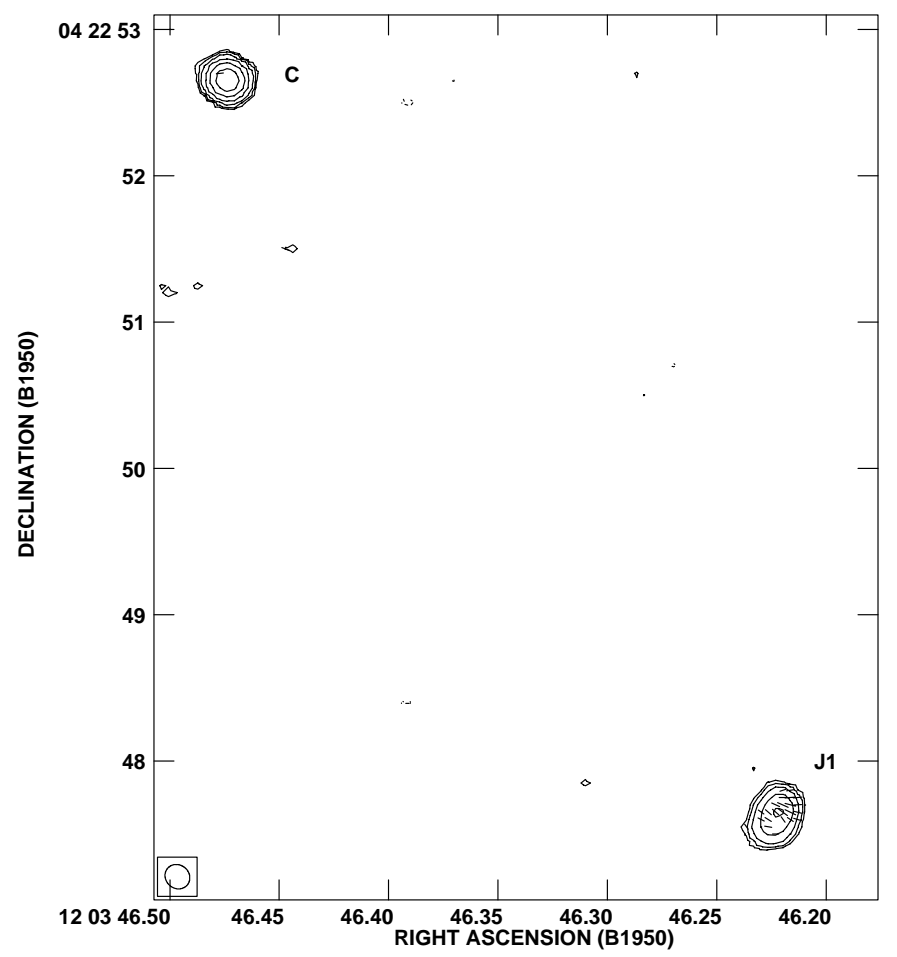

Fig. 9. VLA image of $1203+043$ at $15 \mathrm{GHz}$. Contours are at $-0.4,0.4,0.6,1,2,4,8,16,32 \mathrm{mJy}_{\text {beam }}{ }^{-1}$. The peak flux density is 14.5 mJy beam $^{-1}$. A vector length of $1^{\prime \prime}=$ 10 mJy beam $^{-1}$

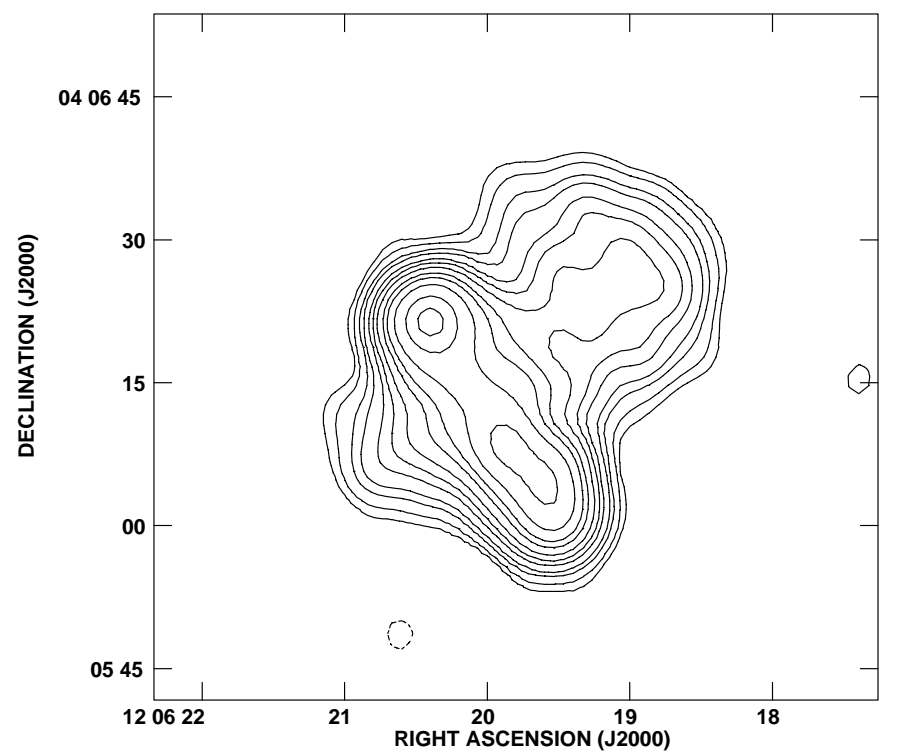

Fig. 10. VLA image of $1203+043$ at $320 \mathrm{MHz}$. Contours are at $-5,5,10,20,30,50,70,100,150,200,300,500,700$, $800 \mathrm{mJy}^{\text {beam }^{-1}}$. The peak flux density is $796 \mathrm{mJy} \mathrm{beam}^{-1}$ component sizes, we conclude that $1203+043$ is likely not variable at low frequencies and that its inclusion in such catalogs is spurious.

Acknowledgements. The authors like to thank the referee, Dr. Steve Spangler for his comments to the paper and Dr. Ian Browne for a critical reading of the manuscript. FM thanks Miller Goss, Assistant Director, NRAO, Socorro, for his hospitality during period when part of the work was done. The National Radio Astronomy Observatory is operated by Associated Universities Inc., under cooperative agreement with the National Science Foundation; AIPS is NRAO's Astronomical Image Processing System.

\section{References}

Altschuler D.R., Broderick J.J., Condon J.J., et al., 1984, AJ 89, 1784

Bondi M., Padrielli L., Gregorini, et al., 1994, A\&A 287, 396

Browne I.A.W., Mantovani F., Muxlow T.W.B., et al., 1985, MNRAS 213, 945

Cechiara P.L., Browne I.A.W., Mantovani F., Muxlow T.W.B., 1994, MNRAS 267, 247

Condon J.J., Cotton W.D., Greisen E.W., et al., AJ 115, 1693

Cotton W.D., 1976, ApJ 204, L63

Ekers R.D., Fanti R., Lari C., Parma R., 1978, Nat 276, 588

Fanti C., Fanti R., Ficarra A., et al., 1983, A\&A 118, 171

Klein U., Mack K.-H., Gregorini L., Parma P., 1995, A\&A 303, 427

Liu R., Pooley G., 1991, MNRAS 253, 669

Mantovani F., Saikia D.J., Browne I.W.A., et al., 1990a, MNRAS 245, 427

Mantovani F., Fanti R., Gregorini, et al., 1990b, A\&A 233, 535

Mantovani F., Junor W., Fanti R., et al., 1992, MNRAS 257, 353

McAdam W.B., 1980, PASA 4, 70

Morganti R., Killeen N.E.B., Tadhunter C.N., 1993, MNRAS 263,1023

Padrielli L., Aller M.F., Aller H.D., et al., 1987, A\&AS 67, 63

Perley R.A., Schwab F.R., Bridle A.H., 1989, Synthesis Imaging in Radio Astronomy Astron. Soc. of the Pacific Conf. Ser. 6, San Francisco

Rickett B.J., 1986, ApJ 307, 564

Sakurai T., Spangler S.R., 1994, Rad. Sci. 29, 635

Spangler S.R., Cotton W.D., 1981, AJ 86, 730

Spangler S.R., Eastman W.A., Gregorini L., et al., 1993, A\&A 267, 213; Erratum, 1994, A\&A 286, 349

Tadhunter C.N., Morganti R., di Serego Alighieri S., et al., 1993, MNRAS 263, 999

Thompson A.R., Clark B.G., Wade C.M., Napier P.J., 1980, ApJS 44, 151

Ulrich-Demoulin M.-H., Rönnback J., 1996, Proceeding of the Symposium IAU 175 "Extragalactic Radio Sources", Ekers R.D., Fanti C., Padrielli L. (eds.). Kluwer Academic Press, p. 254 